\title{
Retinoic Acid Enhances Drug-Induced Cell Death in Anticancer Drug-Resistant Cell Lines
}

\author{
Young Mi Whang, M.S. ${ }^{1}$, Yeul Hong Kim, M.D. ${ }^{1,2,3}$, Sang Won Shin, M.D. ${ }^{1,2}$, Byung Soo Kim, M.D. ${ }^{1,3}$, Jun \\ Suk Kim, M.D. ${ }^{1,3}$, Young Do Yoo, Ph.D. ${ }^{2}$ and Sun-Hee Park, Ph.D. ${ }^{1}$ \\ ${ }^{1}$ Department of Internal Medicine and Division of Brain Korea 21 Program for Biomedical Science, ${ }^{2}$ Genomic Research \\ Center for Lung \& Breast/Ovarian Cancer, ${ }^{3}$ Department of Internal Medicine, Korea University College of Medicine, Seoul, \\ Korea
}

Purpose: Retinoids (RA), a group of vitamin A derivatives, is known to be important for regulation of normal cellular growth and differentiation. RA treatment of various cancers resulted in cell growth inhibition and apoptosis. Therefore, the chemotherapeutic and chemopreventative activities of various types of tumor have been examined. Biological actions of RA are mediated through nuclear receptors, including the retinoic acid receptors (RARs) and retinoid $X$ receptors (RXRs). In this study, we examined the effect of all-trans-retinoic acid (atRA) as an anticancer drug-sensitiser in cancer cell lines and in its drug- resistant cancer cell lines

Materials and Methods: Cells were maintained by RPMI 1640 medium containing $10 \%$ fetal bovine serum. Cells were treated with $1 \mu \mathrm{M}$ atRA for $48 \mathrm{~h}$, then with the desired concentration of anticancer drug for $24 \mathrm{~h}$. Cell viability was measured spectrophotometrically at $540 \mathrm{~nm}$ using the MTT [3-(4,5-dimethylthiazol-2-yl)-2,5-diphenyl tetrazolium bromide] assay. Western blot analyses were performed with the desired antibodies.

Results: We investigated if pre-treatment with atRA

\section{INTRODUCTION}

Radiation therapy and chemotherapy, in conjunction with surgical operations, are commonly employed for the treatment of various types of tumor. However, a significant number of tumors often fail to respond to these therapies, because many forms of tumor appear to be less sensitive, or become resistant, to radiation and anticancer drugs following consecutive treatments. Although the mechanism of resistance to anticancer

Correspondence: Sun-Hee Park, Department of Internal Medicine and Division of Brain Korea 21 Program for Biomedical Science, Korea University College of Medicine, Seoul 136-705, 150050, Korea. (Tel) 02-920-5158, (Fax) 02-926-4534, (E-mail) ydy@ korea.ac.kr

Received November 13, 2001, Accepted May 7, 2002

This work was supported by the Brain Korea 21 Project in 2002. enhanced the drug-sensitivity of various cancer cell lines to either 5-fluorouracil, adriamycin, or cisplatin. 5-FU (SNU638-F2) and CDDP-resistant cell (SNU638-Cis) lines, from a Korean gastric cancer cell line (SNU638) and the ADR-resistant cells (AD600) was established from a colon cancer cell line (SW620). Treatment of each cell line, with $1 \mu \mathrm{M}$ atRA, prior to drug exposure resulted in enhanced cell death in these cell lines. Furthermore, the effect of atRA on growth inhibition, in each drug-resistant cell line, was more obvious than in their parent cell lines. Increased activity of Transglutaminase II (Tgasell) and cleavage of Poly (ADP-ribose) polymerase (PARP) were also observed (western blot analysis

Conclusion: Based on our data, we suggest that atRA enhances anticancer drug-induced cell death and reverses the drug-sensitivity of the drug-resistant cancer cell lines. (Cancer Research and Treatment 2002;34:212-217)

Key Words: Retinoic acid, Drug-resistant cancer cell lines, 5-fluorouracil, Cisplatin, Adriamycin

drugs is not clearly known, it may involve alteration to various molecular processes, including drug influx and efflux, drug metabolism, drug targeting, or the response to cellular damage. It has been reported that resistance to anticancer drugs is caused by induced expression of drug transporters, such as P-glycoprotein (P-gp), a transmembrane drug efflux pump (1). Other work has shown that loss of ceramide production is one cause of cellular resistance to apoptosis, induced by either ionizing radiation or ADR (2). Lavie et al. showed glucosylceramide accumulated the ADR-resistant MCF-7 cell line compared with the drugsensitive MCF-7 cell line, when trying to define the relationship between glycolipids and multidrug resistance (3). Resistance to CDDP has been reported in various mechanisms in tumors and experimental cell culture systems. Some of the mechanisms include: reduced drug accumulation, increased glutathione and metallothioneins preventing the formation of DNA adducts (4), and enhanced repair of damaged DNA by CDDP-induced cytotoxicity (5). Chin $\mathrm{KW}$ et al suggested that a mutant cAMPdependent protein kinase (PKA), with defective regulatory (RIa) 
subunits, exhibit increased resistance to CDDP in Chinese hamster ovaries $(\mathrm{CHO})$ and mouse adenocortical carcinoma cells $(6,7)$. Also, blocking of 5-FU incorporation into nucleic acids, and thymidine kinase overexpression, are responsible for the resistance in 5-FU-resistant cell lines from the Korean gastric cancer cell line (8). Therefore, it is important to overcome resistance to anticancer drugs and develop an anticancer drug-sensitiser for improved drug treatment of cancers.

Retinoids, a group of vitamin A derivatives, are known to be important for regulation of normal cellular growth and differentiation. In addition, they show potential as chemotherapeutic agents in the inhibition of uncontrolled cell growth, inducing apoptosis and differentiation of cancer cells $(9,10)$. It has been suggested that they could play a chemopreventative role in the reversible promotion stage of multistep carcinogenesis. Therefore, their chemotherapeutic and chemopreventative activities, in various types of tumor, have been examined.

atRA, and its isomer 9-cis RA, translocate to the nucleus where they bind to the retinoic acid receptors (RARs) or retinoid $\mathrm{X}$ receptors (RXRs). atRA binds and activates the RARs, whereas 9-cis-retinoic acid binds and activates both the RARs and RXRs. Both receptors are ligand-inducible transregulators that modulate the transcription of the RA target genes by associating with cis-acting RA response elements (RARE). The complex actions of RAR and RXR isotypes $(\alpha, \beta, y)$ mediate the multiple effects of retinoids through the formation of homo or heterodimers (11). Of the isotypes, RAR $\beta$ has been suggested to play an important role in the biological functions of RA in many different types of cancer cells.

RAR $\beta$ expression is also thought to be associated with the cellular sensitivity to retinoid in numerous cancer cells $(12,13)$. Treatment of various types of cancer cells with RA resulted in cell growth inhibition and apoptosis. The mechanism by which RA treatment causes growth inhibition and apoptosis is not clear, but it has been proposed that RA treatment enhances an expression of the cyclin dependent kinase inhibitors, p21 and p27, resulting in cell cycle arrest at the G1 phase in breast, and lung, cancer cells $(14,15)$. Inactivation of cyclin dependent kinases, and the activation of proteins, involved in the apoptotic process were also observed in RA-treated lymphoma cell lines (16). Preliminary clinical results have demonstrated the importance of retinoids in combination chemotherapy of cancer (17). Retinoids may increase the activity of other biological or chemotherapeutic agents, and may overcome tumor resistance to conventional anticancer agents by enhancing cellular cytotoxicity. Therefore, we examined the effect of all trans retinoic acid (atRA) as an anticancer drug-sensitiser in cancer cell lines, and in cancer cell lines resistant to it. We demonstrated a combination treatment, with atRA and anticancer drugs, for the sensitization of drug-resistant cancer cell lines.

\section{MATERIALS AND METHODS}

\section{1) Drugs and chemicals}

3-(4,5-dimethylthiazol-2-yl)-2,5-diphenyl tetrazolium bromide (MTT), and all-trans retinoic acid (atRA) were purchased from Sigma Chemical Co.(St. Louis, MO). 5-fluoruracil (5-FU) and cisplatin (CDDP) were purchased from Korea United Pharm. Inc. (Seoul, South Korea), and adriamycin (ADR) from Dong-A Pharm. Inc. (Seoul, South Korea). All other chemicals were of reagent grade and were purchased from Sigma Chemical Co.

\section{2) Cell lines and cell culture}

The Korean gastric cancer cell lines used in these studies, SNU638, were purchase for KCLB, its 5-FU resistant cell line (SNU638-F2), and cisplatin-resistant cell line (SNU638-Cis), human colorectal adenocarcinoma cell line (SW 620) and its adriamycin-resistant cell line (AD600) were used. These cell lines were cultivated in RPMI 1640 (Gibco/BRL, Grand Island, NY) medium, supplemented with $10 \%$ fetal bovine serum (FBS) and antibiotics, and incubated at $37^{\circ} \mathrm{C}$ under $5 \% \quad \mathrm{CO}_{2}$.

\section{3) Anticancer drug sensitivity}

Cell viability and the growth rate were determined by the 3-(4,5-dimethylthiazol-2-yl)-2,5-diphenyl tetrazolium bromide (MTT)-based colorimetric assay (18). Cells $\left(2 \times 10^{3} \sim 3 \times 10^{3}\right)$ were seeded in 96-well plates with RPMI1640 containing $0.5 \%$ FBS. The cells were treated with various concentrations of anticancer drugs. After $48 \mathrm{~h}$, MTT solution was added to each well, and the plate incubated at $37^{\circ} \mathrm{C}$ for $4 \mathrm{~h}$. Cell viabilities were measured according to the manufacture's instructions, and the absorbance, in individual wells, was determined at $545 \mathrm{~nm}$ using a microplate reader (BioRad, Hercules, CA). The $\mathrm{IC}_{50}$ value was determined by calculation from the dose-effect analysis using a microcomputer (Biosoft, Cambridge, UK).

\section{4) All-trans retinoic acid treatment and cytotoxicity assay}

Cells were grown in 96-well plates starting at an initial density of $2 \times 10^{3} \sim 3 \times 10^{3}$ cells/well. $24 \mathrm{~h}$ prior to RA treatment, the culture medium was changed to RPMI 1640 containing $0.5 \%$ FBS, to minimise the effect of endogenous RA in the culture medium. $1 \mu \mathrm{M}$ atRA, solubilized in DMSO, was added to the medium, and the cells grown for a further days, depending on the experimental conditions. $0.01 \%$ DMSO, used to control the RA, did not affect the proliferation of the cells tested. After $48 \mathrm{~h}$, following treatment with $1 \mu \mathrm{M}$ atRA, they were treated with the desired anticancer drug. $3 \mathrm{~h}$ after this treatment, the culture medium was changedto RPMI1640 medium containing the anticancer drug, and incubated for a further $48 \mathrm{~h}$. Cell viability was measured spectrophotometrically at $540 \mathrm{~nm}$ using the MTT assay.

\section{5) Protein extraction and western blot analysis}

Cells were plated on $60 \mathrm{~mm}^{2}$ culture dishes with $5 \times 10^{4}$ cells $/ \mathrm{cm}^{2}$. The cells were harvested and counted, following treatment with atRA and the anticancer drug, as described. atRA and anticancer drug-treated cells were harvested by centrifuging at $12,000 \mathrm{rpm}, 4^{\circ} \mathrm{C}$ for 10 minutes, and washed with ice-cold phosphate buffered saline (PBS, pH 7.4). The cells were lysed with 50jl of ice-cold RIPA buffer $(10 \mathrm{mM}$ Tris- $\mathrm{HCl}, \mathrm{pH} 7.4,150 \mathrm{mM} \mathrm{NaCl}, 1 \%$ sodium deoxycholate, $1 \%$ NP-40, 0.1\% SDS, $1 \mathrm{mM}$ PMSF, $1.5 \mu \mathrm{g} / \mathrm{ml}$ aprotinin and $2 \mu \mathrm{g} /$ $\mathrm{ml}$ leupeptin), and the supernatant stored at $70^{\circ} \mathrm{C}$ until needed. Total cell lysates $(30 \sim 50 \mu \mathrm{g})$ were resolved on $8 \sim 12 \%$ SDSPAGE gel and transferred to PVDF membranes (Amersham Pharmacia Piscataway, NJ). The blots were probed with ant- 
TGasell (NeoMarker, Fremont, CA), anti-PARP (Pharmingen, A Becton Dickinson, Franklin Lakes, NJ), anti-bcl-2 (Pharmingen), caspase-3, caspase-9 (Santa Cruz Biotechnology, Santa Cruz, CA), and anti- $\beta$-actin (BIODESIGN Co., Saco, $\mathrm{MN})$, according to the manufacturer's instructions.

\section{6) Statistical analysis}

The $\mathrm{IC}_{50}$ values are shown as the mean \pm SD. Significant differences between the values were determined by classical two-way ANOVA tests. All cases where the $p$-values were less than 0.05 were considered statically significant.

\section{RESULTS}

\section{1) Sensitivity to anticancer drugs}

Fig. 1 shows the cytotoxicity of cancer cell lines treated with various concentrations of each anticancer drug. Drug-resistant cell lines showed an acquired resistance to 5-FU, CDDP and ADR compared with drug-sensitive cell lines. SNU638-F2 exhibited strong resistance to 5-FU compared with SNU638. They were more resistant to CDDP and ADR in SNU638-Cis and AD600 than in SNU638 and SW620. We also compared the anticancer drugs-induced growth inhibition of drug-sensitive, and drug-resistant, cell lines, as shown in Table 1.

\section{2) Inhibition of cell growth by the combination of atRA and anticancer drugs}

In Fig. 2, the results of the combinational studies are shown. Cell lines were treated with the desired concentration of anticancer drug, after pretreatment with $1 \mu \mathrm{M}$ atRA, as described in materials and methods. 5-FU and CDDP doses were fixed at $5 \mu \mathrm{g} / \mathrm{ml}$, whereas, the ADR concentration was $2 \mu \mathrm{g} /$ $\mathrm{ml}$. 5-FU alone increased the cytotoxicity by $80 \%$ in SNU638 cell line, but had no effect on the SNU638-F2 cell line. A single treatment of atRA was ineffective on the cytotoxicity of both cell lines. However, combined treatment with atRA and 5 -FU increased the cytotoxicity by more than $20 \%$ in the SNU638-F2 cell line (Fig. 2A). As a result, we conclude that treatment with either atRA or 5-FU alone does not induce cytotoxicity in the 5-FU-resistant cell line. However, combination of treatment, with atRA and 5-FU, shows a synergistic effect.

Fig. 2B shows the synergistic effect of atRA and CDDP. When the cells were treated with CDDP only, it showed $45 \%$
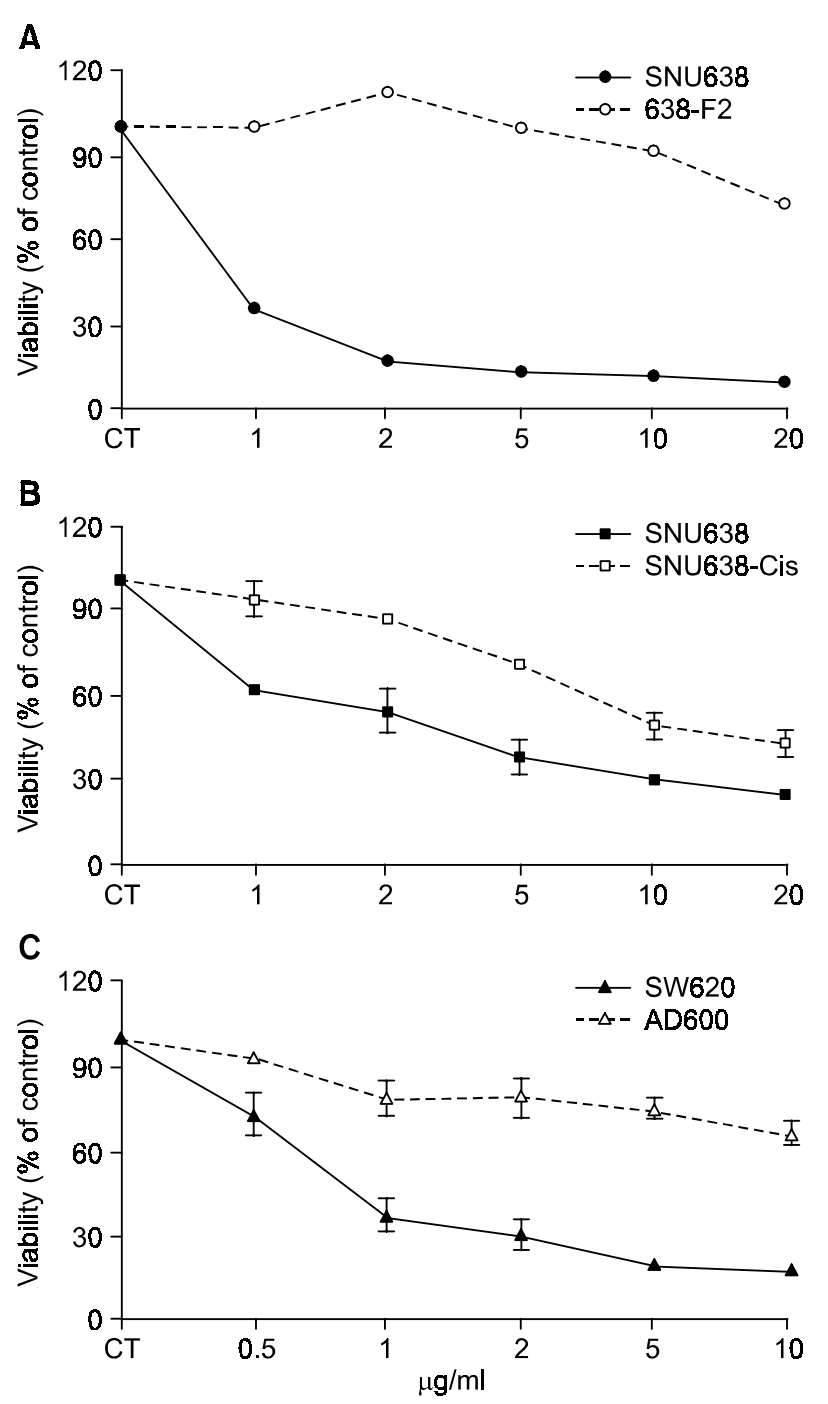

Fig. 1. Cytotoxicity of anticancer drugs in SNU638 and SW620 cell lines. SNU638, SW620 and their anticancerdrugresistant cell lines (SNU638-F2, SNU638-Cis and AD600) were treated with each anticancer drug for $24 \mathrm{~h}$. Percent cell viability was calculated, compared to control (closed bars: parent cell lines, open bars: drug-resistant cell lines).

Table 1. Comparison of drug resistance of SNU638, 638-F2, 638-Cis, SW620 and AD600 cells

\begin{tabular}{|c|c|c|c|c|c|}
\hline Anticancer drugs & 5-Fluorouracil & \multicolumn{2}{|c|}{ Cisplatin } & \multicolumn{2}{|c|}{ Adriamycin } \\
\hline Cell lines & SNU638 638-F2 & SNU638 & 638-Cis & SW620 & AD600 \\
\hline $\mathrm{IC}_{50} *(\mathrm{\mu g} / \mathrm{ml})$ & $0.113 \quad 25.257$ & $1.96 \pm 0.01$ & $12.4 \pm 82.6$ & $0.96 \pm 0.3$ & $33.27 \pm 24.5$ \\
\hline $\mathrm{RR}^{\dagger}$ & 223.5 & \multicolumn{2}{|l|}{6.4} & \multicolumn{2}{|c|}{34.7} \\
\hline
\end{tabular}

*drug concentration $(\mathrm{\mu g} / \mathrm{ml})$ at which cell growth is inhibited by $50 \%,{ }^{\dagger}$ relative resistance $-\mathrm{IC}_{50}$ for resistant cell line/IC $\mathrm{C}_{50}$ for parental cell line 
A
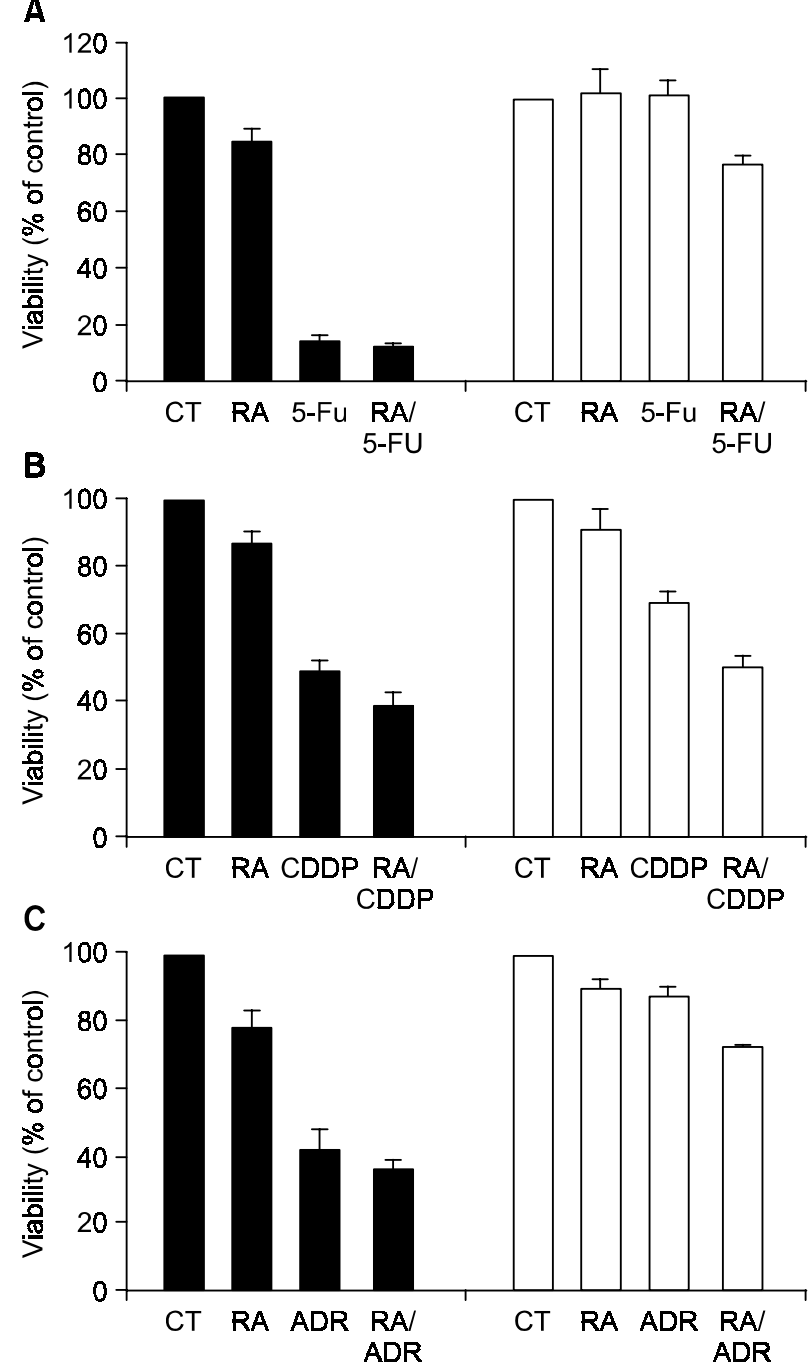

Fig. 2. Effect of atRA on cytotoxicity of anticancer drugs in SNU638 and SW620 cell lines. SNU638, SW620 and their anticancerdrug-resistant cell lines (SNU638-F2, SNU 638-Cis and AD600) were treated with each anticancer drug for $24 \mathrm{~h}$, then were treated with $1 \mathrm{\mu M}$ atRA for 24 h. Percent cell viability were calculated, compared to control. The treatment concentrations of anticancer drugs were; $5 \mathrm{\mu g} / \mathrm{ml}$ for $5-\mathrm{FU}$ and $\mathrm{CDDP}, 2 \mathrm{\mu g} / \mathrm{ml}$ for ADR. (A) SNU638 and SNU638-F2 cells, (B) SNU638 and SNU638-Cis cells, (C) SW620 and AD600 cells.

of cell viability in SNU638 and 75\% in SNU638-Cis. Treatment with atRA alone was ineffective in the growth inhibition of both cell lines. In combination with CDDP, atRA pretreatment showed only an $8 \%$ increase of cytotoxicity in SNU638 cells, compared to treatment with CDDP only. However, in SNU638-Cis cells, atRA pretreatment showed increased growth inhibition of $30 \%$.

When ADR was administered at a dose of $2 \mu \mathrm{g} / \mathrm{ml}$, following pre-treatment with atRA for $48 \mathrm{~h}$, we observed the same result in the human colorectal adenocarcinoma, and ADR-resistant, SW620 cell lines (Fig. 2C). atRA alone showed 90\% cell viability in both cell lines. When ADR was treated alone, the cell viability was $45 \%$ in SW620 and $86 \%$ in AD600. The AD600 cell line displayed strong resistance to atRA and ADR. In spite of the strong resistance to atRA and ADR, the combinational treatment with atRA and ADR showed increased cytotoxicity of $30 \%$.

3) Western blot analysis of the apoptosis-related proteins

To determine if increased cytotoxicity is related in apoptosis, we performed Western blot analyses. Fig. 3A shows that the typical $M_{\mathrm{r}} 85,000(85 \mathrm{kDa})$ cleavages of PARP were increased in 5-FU-treated cells following pretreatment with atRA, especially in the SNU638-F2 cell line. Cleavage of PARP was not detected in the untreated control of SNU638-F2. Expression of TGase II had no effect on treatments with either agent. Interestingly, expression of $\mathrm{Bcl}-2$ was distinctly reduced in atRA and 5-FU-treated cells. It seems that apoptosis is responsible for the effective growth inhibition with atRA and 5-Fu. Fig. 3B demonstrates that expression of TGaseII did not alter either cell line, whereas the increased cleavage of PARP was more obvious in SNU638-Cis than in the parental cells, when the cells were treated with atRA and CDDP in combination. In the case of ADR-resistant SW620 cells, the expression of TgaseII was not affected by treatment with atRA and ADR (Fig. 3C). Cleavage of PARP was induced in ADR-treated and atRA and ADR-treated cells, to a small degree.

\section{DISCUSSION}

Acquired resistance to anticancer-drugs following consecutive chemotherapy becomes a serious obstacle in cancer treatment. Although there are individual differences for different types of tumor in term of sensitivity to anticancer drugs, many types of tumor are less sensitive, or become resistant, to anticancer drugs. Therefore, in this study, we investigated a combination treatment of atRA alone, with anticancer drugs, to try and establish a strategy were by atRA treatment could be used to recover the sensitivity to drug-resistance. We demonstrated that pretreatment with atRA enhanced the cytotoxicity of cancer cells to low doses of anticancer drugs. We further investigated atRA treatment to determine if it played the role of a sensitiser of anticancer drugs, in terms of drug-induced cytotoxicity, in solid tumors.

In order too visibly maximise the effect of a combinational treatment, we first determined a treatment dosage of anticancer drugs, based on our preliminary experimental data (data not shown). We found this caused less than $50 \%$ toxicity of cancer cells, to each drug in drug-sensitive cell lines, and less than $10 \%$ in drug-resistant cell lines.

Treatment with atRA and anticancer drugs alone, had no affect on cell growth inhibition in drug-resistant cell lines, per se, resulting in a growth inhibition of less than $10 \%$. However, a combinational treatment, of atRA with anticancer drugs, significantly increased the cytotoxicity of anticancer drugs in the SNU638-F2 cell line by $20 \%$, and by approximately $30 \%$ in SNU638-Cis and AD600 cell lines (Fig. 2). These data suggest the combinational treatment, with atRA and anticancer drugs, synergistically affect the cytotoxicity of cancer cell lines, especially drug-resistant cell lines. Various combinational treat- 


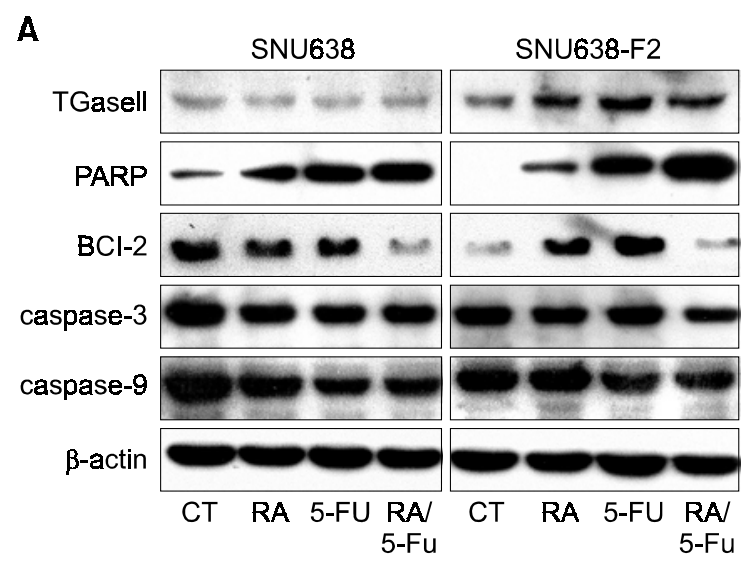

B

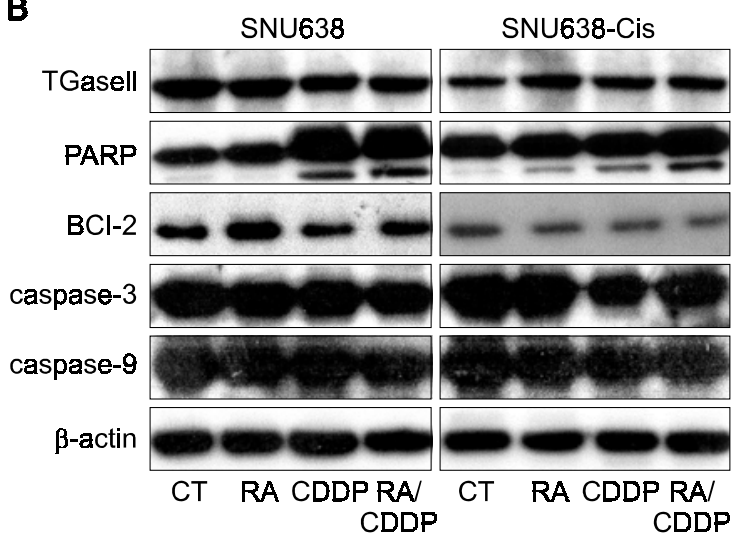

C

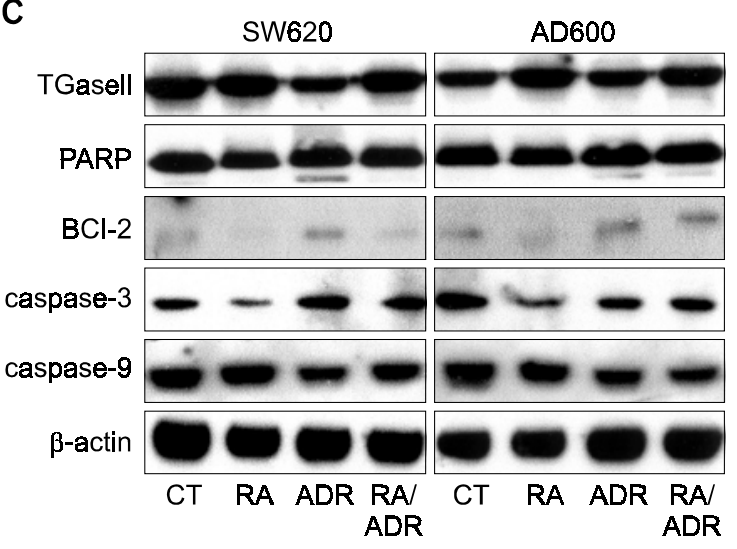

Fig. 3. Expression of proteins related with apoptosis in SNU638 and SW620 cell lines. SNU638, SW620 and their anticancerdrug-resistant cell lines (SNU638-F2, SNU638-Cis and AD600) were treated as mentioned in Fig. 2 and cells were harvested for western blots described in Methods and Materials. (A) SNU638 and Snu638-F2 cells, (B) SNU638 and SNU638-Cis cells, (C) SW620 and AD600 cells.

ments have been tried for the purpose of improving the drug sensitivity of conventional anticancer drugs in cancer cell lines. Combinational treatments with atRA and cytokines, or anticancer drugs, have shown synergistic antitumor effects in a various human cancer cell lines, such as human breast, ovarian and squamous head and neck, cancer cell lines, as well as malignant melanomas $(15 \sim 17)$. In this study, we first demonstrated the function of atRA as an enhancer of drug sensitivity in anticancer drug-resistance cancer cell lines.

From our preliminary data, we found that the treatment order of combinational drugs was important in increasing the cytotoxicity of anticancer drugs (data not shown). atRA pretreatment, prior to treatment of an anticancer drug, caused a synergistic increase in cytotoxicity of combination treatments in cancer cells. However, treatment of cells with atRA, after anticancer drug treatment, atRA appeared to protect cells from oxidative stress caused by anticancer drugs, presumably due to its antioxidant activity (18).

Apoptosis is the process of cell death, resulting in substrate cleavage of intracellular polypeptides, by a protease known as caspases (19). PARP is cleaved by caspases when apoptosis occurs. In our data, combinational treatment with atRA and anticancer drugs, increased cleavage of PARP in drug-resistant cell lines, compared to the untreated control (Fig. 3). Apparently, atRA treatment of cells results in decreased expression of caspase-3, especially in SW620 and AD600 cell lines. A 35 $\mathrm{kDa}$ inactive form of caspase- 3 was cleaved by atRA treatment. It seems that the cleavage of caspase- 3 may cause a proteolytic activation of the molecules. However, atRA treatment caused no cleavage of caspase 3 in other cell lines. In all cancer cell lines examined in our research the cleavage of $116 \mathrm{kDa}$ PARP to the $85 \mathrm{kDa}$ fragment was observed during treatment with the atRA or anticancer drug alone, and in combination. The $85 \mathrm{kDa}$ fragment was predominantly increased with combinational treatment, especially in SNU638-F2 and SNU638-Cis cancer cells treated with atRA and either 5-FU or CDDP.

Tissue transglutaminase type II (TGaseII) has been reported as a differentiation, and apoptosis, marker (20). TGase activity has been shown to be associated with the effects of retinoic acid (RA) in various cell types $(21 \sim 23)$. Antonyak MA et al. reported that pretreatment of HL60 and NIH3T3 cells with RA resulted in TGase expression and activation, prior to any inhibitory effects of the atRA on cells (24). In our data, Tgase II expression was increased only in the AD600 cell line, which was induced by atRA pretreatment. Furthermore, an increase in 
the expression of Tgase II occurred related with cleavage of PARP. Therefore, it suggests that atRA pretreatment enhanced the expression of TGase II, which then brought about the cell apoptosis process in combinational drug-treated cancer cells.

Bcl-2 family members are known to be regulators of the apoptosis process. Overexpression of Bcl-2 shows a connection in prostate cancer in developing resistance to the cytotoxic effects of doxorubicin (25). We observed that expression of Bcl-2 decreased when cells were treated with atRA in both SW620 and AD600 cell lines. This result suggests that atRA treatment down-regulated expression of Bcl-2, and contributed to inhibit cellular growth, in the both cell lines. Combinational treatment with atRA and 5-FU also diminished the expression of Bcl-2 in the atRA-treated SNU638-F2 cell line. Combinational treatment with atRA and anticancer drugs showed a different response in the cytotoxicity of cells, depend on the cell type, and the mechanism causing cellular cytotoxicity.

\section{CONCLUSIONS}

From our results, we conclude that treatment with either atRA or 5-FU alone, did not induce cytotoxicity in 5-FUresistant cell lines. The 5-FU resistant SNU638-F2 cell line had a synergistic effect in combination with atRA and 5-FU.

Our data suggests, atRA pretreatment could reverse anticancer drug-resistance, and enhance drug-sensitivity in drugresistance cancer cells. Although atRA treatment has not been tried in vivo, our results are encouraging for the use of atRA as an anticancer drug-sensitiser.

\section{REFERENCES}

1. Juranka P, Zastawny RL, Ling V. P-glycoprotein: multidrugresistance and a superfamily of membrane-associated transport proteins. FASEB J 1989;3(14):2583-2529.

2. Liu YY, Han TY, Giuliano, AE, Hansen N, Cabot MC. Uncoupling ceramide glycosylation by transfection of glucosylceramide synthase antisense reverses adriamycin resistance. J Biol Chem 2000;275:7138-7143.

3. Lavie Y, Cao H, Bursten SL, Giuliano AE, Cabot MC. Accumulation of glucosylceramides in multidrug-resistant cancer cells. J Biol Chem 1996;271:19530-19536.

4. Godwin AK, Meister A, O'Dwyer PJ, Huang CS, Hamilton TC, Anderson ME. High resistance to cisplatin in human ovarian cancer cell lines is associated with marked increase of glutathione synthesis. Proc Natl Acad Sci USA 1992;89:30703074.

5. Eastman A, Schulte N. Enhanced DNA repair as a mechanism of resistance to cis-diamminedichloroplatinum (II). Biochemistry 1988;27:4730-4734.

6. Cvijic ME, Yang WL, Chin KV. Cisplatin resistance in cyclic AMP-dependent protein kinase mutants. Pharmacol Ther 1998; 78(2):115-28. Review.

7. Mary Ellen, Khew-Voon Chin. Effects of RIa overexpression on cisplatin sensitivity in human ovarian carcinoma cells. Biochem Biophys Res Commun 1998;249:723-727.

8. Chung YM, Park SH, Park JK, Kim YT, Kang YK, Yoo YD. Establishment and characterization of 5-fluorouracil-resistant gastric cancer cells. Cancer Lett 2000;159:95-101.

9. R Lotan. Retinoids as modulators of tumor cells invasion and metastasis. Semin Cancer Biol 1991;2:197-208.

10. Hofmann SL. Retinoids-'differentiation agents' for cancer treatment and prevention. Am J Med Sci 1992;304:202-213.

11. Lowe NJ, Marks R. Retinoids: A clinician's guide. $2^{\text {nd }}$ edition London: Martin Dunitz. 1998, pp1-32.

12. Wan H, Oridate N, Lotan D, Hong WK, Lotan R. Overexpression of retinoic acid receptor beta in head and neck squamous cell carcinoma cells increases their sensitivity to retinoid-induced suppression of squamous differentiation by retinoids. Cancer Res 1999;59(14):3518-3526.

13. Sun SY, Wan H, Yue P, Hong WK, Lotan R. Evidence that retinoic acid receptor beta induction by retinoids is important for tumor cell growth inhbition. J Biol Chem 2000;275(22): 17149-17155.

14. Zhu WY, Jones CS, Kiss A, Matsukuma K, Amin S, De Luca LM. Retinoic acid inhibition of cell cycle progression in MCF-7 human breast cancer cells. Exp Cell Res 1997;234(2): 293-299.

15. Hsu SL, Hsu JW, Liu MC, Chen LY, Chang CD. Retinoic acid-mediated G1 arrest is associated with induction of p27 (Kip1) and inhibition of cyclin-dependent kinase 3 in human lung squamous carcinoma CH27 cells. EXP. Cell Res 2000; 258(2):322-331.

16. Dierov J, Sawaya BE, Prosniak M, Gartenhaus RB. Retinoic acid modulates a bimodal effect on cell cycle progression in human adult T-cell leukemia cells. Clin Cancer Res 1999; 5(9):2540-2547.

17. Wang Q, Yang W, Uytingco MS, Christakos S, Waiederl R. 1,25-Dihydroyvitamin D3 and all-trans retinoic acid sensitize breast cancer cells to chemotherapy-induced cell death. Cancer Res 2000;60:2040-2048.

18. Ahlenmeyer B, Bauerbach E, Plath M, Steuber M, Heers C, Tegtmeier F, Krieglstein J. Retinoic acid reduces apoptosis and oxidative stress by preservation of SOD protein level. Free Radic Biol Med 2001;30:1067-1077.

19. Watson WG, Rotstein OD, Parodo J, Bitar R, Hackam D, Marshall JC. Granulocytic differentiation of HL-60 cells results in spontaneous apoptosis mediated by increased caspase expression. FEBS Lett 1997;412:603-609.

20. Chiantore MV, Giandomenico V, De Luca LM. Carcinoma cell lines resistant for growth inhibition and apoptosis to retinoic acid ar responsive to 4-hydroxy-phenyl-retinamide; Correlation with tissue transglutaminase. Biochem Biophys Res Commun 1999;254:636-641.

21. Chiocca EA, Davies PJ, Stein JP. The molecular basis of retinoic acid action. Transcriptional regulation of tissue transglutaminase gene expression in macrophages. J Biol Chem 1988;263:11584-11589.

22. Sporn MB, Roberts AB. Role of retinoids in differentiation and carcinogenesis. Cancer Res 1983;43:3034-3040.

23. Piacentini M, Martinet N, Beninati S, Folk JE. Free and protein-conjugated polyamines in mouse epidermal cells. Effect of high calcium and retinoic acid. J Biol Chem 1988;263:379037904.

24. Antonyak MA, Singh US, Lee DA, Boehm JE, Combs C, Zgola MM, Page RL, Cerione RA. Effects of tissue transglutaminase on retinoic acid-induced cellular differentiation and protection against apoptosis. J Biol Chem 2001;276:33582-33587.

25. Tu SM, McConnell K, Marin MC, Campbell ML, Fernandez A, von Eschenbach AC, McDonnell TJ. Combination adriamycin and suramin induce apoptosis in bcl-2 expressing prostate carcinoma cells. Cancer Lett 1995;93:147-155. 